\title{
BOUNDARY CONTROL OF SOME INFINITE DIMENSIONAL SYSTEMS
}

\author{
Ömer Morgül \\ Bilkent University, Dept. of Electrical and Electronics \\ Engineering, 06800, Bilkent, Ankara, Turkey
}

\begin{abstract}
We will consider the feedback stabilization of a class of in£nite dimensional systems by using boundary control, i.e. control inputs are applied at the boundaries of such systems. Such systems usually possess an internal energy, and along their solutions a conservation of energy equation hold. By utilizing this relation, we will prove various stability results. We will also give an example on the application of the proposed technique to some well known passive systems. We will also present some simulation results.
\end{abstract}

\section{Copyright $(2005$ IFAC}

Keywords: Boundary Control, Infnite Dimensional Systems, Passivity.

\section{INTRODUCTION}

Many mechanical systems, such as spacecraft with aexible attachments, or robots with Qexible links, and many practical systems such as power systems, and mass transport systems contain certain parts whose dynamic behaviour can be rigorously described only by partial differential equations (PDE). In such systems, to achieve high precision demands, the dynamic effect of the system parts whose behaviour are described by PDE's on the overall system has to be taken into account in designing the controllers.

In recent years, boundary control of systems represented by PDE's has become an important research area. This idea is erst applied to the systems represented by the wave equation (e.g. elastic strings, cables), see e.g. (Chen, 1979), and then extended to beam equations, (Chen et. al., 1987), ( Morgül, 1992) and to the rotating aexible structures, see ( Morguil, 1990), (Morgül, 1991). In particular, it has been shown that for a string which is clamped at one end and is free at the other end, a single non-dynamic boundary control applied at the free end is suffcient to exponentially stabilize the system, see e.g. (Chen, 1979). For an extension of these ideas to dynamic boundary controllers, see ( Morgül, 1992), ( Morgül, 1994). For more references on the subject the reader is referred to (Lions, 1988), (Luo, Guo and Morgül, 1999).

While the stabilization is an important subject in its own right, it could also be viewed as a £rst step in designing controllers to achieve some additional tasks such as tracking, disturbance rejection, robustness, etc. In this sense, when a system to be controlled is given, it would be desirable to determine a relatively large class of stabilizing controllers, if possible all. Then within this class of controllers one may try to £nd suitable ones to solve additional problems like tracking, disturbance rejection, etc.

In this work we will consider the boundary control of a class of passive infnite dimensional systems, see (Luo, Guo and Morgül, 1999). We will develop some general results for the stabilization of this class of infnite dimensional systems by means of boundary control techniques. In this class of systems the inputs and outputs are assumed to act on the boundaries of the system. For this class of systems, we will frst investigate the effect of a simple feedback law and prove certain stability results. It can easily be shown that some of the examples frequently encountered 
in the literature (e.g. the wave equation, the EulerBernoulli and the Timoshenko beam equations) can be viewed in this class and we present the stability results for such systems. Various generalizations of the control law mentioned above which may yield similar stability results is also possible. We will follow the framework given in (Luo, Guo and Morgül, 1999).

\section{A GENERAL FRAMEWORK}

To motivate the concept of passivity, let $\mathscr{S}$ be a dynamical system, let $u, y \in \mathbf{R}^{m}$ be its input and output vectors, respectively, let $X$ be a Hilbert space in which the solutions of $\mathscr{S}$ evolve and let $E: X \rightarrow \mathbf{R}$ be an appropriate "energy" function which depends on the solutions of $\mathscr{S}$. Assume that the following holds

$$
\frac{d E}{d t}=u^{T} y=\sum_{i=1}^{m} u_{i} y_{i}
$$

where the derivative is taken along the solutions of $\mathscr{S}$ and we set $u=\left(u_{1} \ldots u_{m}\right)^{T}, y=\left(y_{1} \ldots y_{m}\right)^{T} \in \mathbf{R}^{m}$, the superscript $T$ denotes the transpose. In such systems, $E$ may be called as the internal "energy" of the system and (1) may be viewed as the conservation of energy, where the right hand side of (1) may be viewed as the "external power" supplied to the system, and the left hand side may be viewed as "internal power". Hence, we may also view (1) as a "balance of power" equation. In such a case a natural choice for the control inputs $u_{i}$ for the stabilization is

$$
u_{i}=-\alpha_{i} y_{i}, \quad \alpha_{i} \geq 0,
$$

and if we use (2) in (1), the latter becomes

$$
\frac{d E}{d t}=-\sum_{i=1}^{m} \alpha_{i}\left(y_{i}\right)^{2} .
$$

Hence the control law given by (2) results in the dissipation of "internal energy" of the system, and under appropriate assumptions some stabilization results may be deduced.

To elaborate further, let $H$ be a Hilbert space, let $<\cdot, \cdot\rangle_{H}$ and $\|\cdot\|_{H}$ denote the inner-product and the associated norm for $H$, respectively. Consider the following second order systems:

$$
w_{t t}+A w=0
$$

where a subscript denotes the partial derivative with respect to the corresponding variable, and $A$ is a linear (not necessarily bounded) operator on $H$. Assume that $A$ depends on the (one dimensional) spatial variable $x$ and that $x \in[0,1]$. Assume that the system given by (4) has the following boundary conditions

$$
\begin{aligned}
& \left(B_{i}^{1} w\right)(0)=f_{i}^{1}, i=1, . ., k \\
& \left(B_{i}^{2} w\right)(1)=f_{i}^{2}, i=1, . ., l
\end{aligned}
$$

$$
\begin{aligned}
& \left(B_{i}^{3} w\right)(0)=0, i=1, . ., p \\
& \left(B_{i}^{4} w\right)(1)=0, i=1, . ., r
\end{aligned}
$$

where $B_{i}^{j}$ are various linear (not necessarily bounded) operators on $H, k, l, p, r$ are some appropriate integers, and $f_{i}^{j}$ are control inputs of our systems. In the sequel we will not state the range of indices, which should be obvious from the context. We note that here $\left(B_{i}^{j} w\right)(\cdot):[0,1] \rightarrow H$ and $\left(B_{i}^{j} w\right)(c)$ denotes the value of $B_{i}^{j} w$ at $x=c$.

Let us defne the following sets

$$
\begin{gathered}
\mathscr{S}_{1}=\left\{w \in H \mid\left(B_{i}^{1} w\right)(0)=0,\right. \\
\left.\left(B_{i}^{2} w\right)(1)=0\right\}, \\
\mathscr{S}_{2}=\left\{w \in H \mid\left(B_{i}^{3} w\right)(0)=0, .\right. \\
\left.\left(B_{i}^{4} w\right)(1)=0\right\}
\end{gathered}
$$

Let $D(A) \subset H$ be the domain of $A$. For simplicity we may take

$$
D(A)=\{w \in H \mid A w \in H\} .
$$

Let $A_{u c}$ denote the operator $A$ with the following domain

$$
D\left(A_{u c}\right)=D(A) \cap \mathscr{S}_{1} \cap \mathscr{S}_{2}
$$

We make the following assumptions

Assumption 1 : $\mathrm{D}(\mathrm{A})$ is dense in $H$.

Assumption $2: D\left(A_{u c}\right)$ is dense in $H, A_{u c}$ is selfadjoint and coercive in $H$, i.e. the following holds for some $\alpha>0$

$$
<w, A_{u c} w>_{H} \geq \alpha\|w\|_{H}^{2}, w \in D\left(A_{u c}\right) .
$$

From the Assumption 2 it follows that $A_{u c}^{1 / 2}$ exists, is self-adjoint and nonnegative. We will de£ne the set $V$ as

$$
V=D\left(A_{u c}^{1 / 2}\right)
$$

For the set $V$, we make the following assumption for technical reasons.

Assumption 3 : The set $V \subset H$ satis£es the following

$$
V \cap \mathscr{S}_{1} \neq V, V \cap \mathscr{S}_{2}=V .
$$

We note that in most of the cases, the sets $\mathscr{S}_{1}$ and $\mathscr{S}_{2}$ impose certain conditions on $w \in H$ at the boundaries, and the set $V$ could be rede£ned without changing the density arguments so that the Assumption 3 is satis£ed.

Let us consider the system given by (4)-(6) with $f_{i}^{1}=$ $f_{i}^{2}=0$ for $i=1, \ldots, k, l$, whichever appropriate. The resulting system is called uncontrolled since the control inputs are set to zero. We can rewrite (4) as

$$
\frac{d z}{d t}=\mathscr{A} z, z(0) \in X
$$


where $X=V \times H, z=\left(w w_{t}\right)^{T} \in X$, and $\mathscr{A}$ is a linear operator defned on $X$ as

$$
\mathscr{A}=\left[\begin{array}{cc}
0 & I \\
-A & 0
\end{array}\right],
$$

with $D(\mathscr{A})=D\left(A_{u c}\right) \times V$. Here, and in the sequel, the superscript $T$ denotes the transpose. For $z_{1}=$ $\left(u_{1} v_{1}\right)^{T}, z_{2}=\left(u_{2} v_{2}\right)^{T} \in X$, the inner-product and the norm on $X$ is defned as

$$
\begin{gathered}
<z_{1}, z_{2}>_{X}=<A_{u c}^{1 / 2} u_{1}, A_{u c}^{1 / 2} u_{2}>_{H} \\
+<v_{1}, v_{2}>_{H}, \\
\|z\|_{X}^{2}=\left\|A_{u c}^{1 / 2} u\right\|_{H}^{2}+\|v\|_{H}^{2},
\end{gathered}
$$

where $z=(u v)^{T} \in X$.

Consider the system given by (4)-(6). Our aim is to £nd control laws for $f_{i}^{j}$ such that the resulting system possesses the following properties :

i : There exists a solution to (4)-(6) in an appropriate space and this solution is unique (well-posedness problem),

ii : The solution of (4)-(6) decays to zero as $t \rightarrow \infty$ (asymptotic stability problem).

In the sequel we will propose a class of feedback control laws to solve the problems posed above. In such feedback schemes, the control inputs are appropriate functions of $w$ and/or $w_{t}$, evaluated at appropriate boundary. Such functions are naturally called as the outputs of the system. The selection of appropriate outputs are necessary for the control schemes based on passivity and our next assumption clarifes this point.

Assumption 4 : Let $D_{1}=D(A) \cap \mathscr{S}_{2}$ and $D=D_{1} \times V$. $D_{1}$ is dense in $D\left(A_{u c}\right)$ and the following holds

$$
\begin{aligned}
<z, \mathscr{A} z\rangle_{X}= & \sum_{i=1}^{k}\left(B_{i}^{1} u\right)(0)\left(O_{i}^{1} v\right)(0) \\
& +\sum_{i=1}^{l}\left(B_{i}^{2} u\right)(1)\left(O_{i}^{2} v\right)(1),
\end{aligned}
$$

where $z=(u v)^{T} \in D$ and $O_{i}^{j}, i=1, \ldots k$ or $l, j=$ 1,2 , whichever appropriate, are linear (not necessarily bounded) operators on $H$. We will call (18) as the power form for the system given by (14). (cf. (1)).

Remark 1 : Let us defne the energy $E(t)$ of the solutions of (14) as

$$
E(t)=\frac{1}{2}<z(t), z(t)>_{X} .
$$

By differentiating (19), by noting that $z(t)$ is a solution of (14), hence $f_{i}^{j}=0$, and by using (18), we obtain $d E / d t=0$, i.e. the energy is conserved for the uncontrolled case. We will choose the control inputs appropriately by using the power form given by (18) so that the energy is dissipated and all solutions asymptotically decay to zero.
Let $z=\left(w w_{t}\right)^{T}$ be the solution of (14). By considering (18), we de£ne the outputs $y_{i}^{j}$ of the system (14) as

$$
\begin{gathered}
y_{i}^{1}=\left(O_{i}^{1} w_{t}\right)(0), i=1, . ., k, \ldots \\
y_{i}^{2}=\left(O_{i}^{2} w_{t}\right)(1), i=1, . ., l
\end{gathered}
$$

Let us consider the system given by (4)-(6) and assume that the Assumptions 1-4 hold. Here the $f_{i}^{j}$ are the inputs and the outputs are chosen as in (20). We will denote the resulting system as $\mathscr{S}$. In this framework, the power form given by (18) takes the following form

$$
<z, \mathscr{A} z>_{X}=\sum_{i=1}^{k} f_{i}^{1} y_{i}^{1}+\sum_{i=1}^{l} f_{i}^{2} y_{i}^{2}
$$

For the system $\mathscr{S}$, the control problem we consider can be stated as follows : Find appropriate control laws for $f_{i}^{j}$ by using the outputs $y_{i}^{j}$ such that the resulting closed-loop system is well-posed and asymptotically stable. While it is possible to use a general controller which relates the set of outputs to the set of inputs, here we will consider a simple choice in which $f_{i}^{j}$ is related only to $y_{i}^{j}$ as follows

$$
f_{i}^{j}=-\alpha_{i}^{j} y_{i}^{j}
$$

where $\alpha_{i}^{j} \geq 0$, (cf. (2)). Such a selection is quite natural when we consider the power form (21) which becomes the following by using (22)

$$
<z, \mathscr{A} z>_{X}=-\sum_{i=1}^{k} \alpha_{i}^{1}\left(y_{i}^{1}\right)^{2}-\sum_{i=1}^{l} \alpha_{i}^{2}\left(y_{i}^{2}\right)^{2} .
$$

Hence $\mathscr{A}$ becomes dissipative with this controller. This property is of crucial importance in proving both the well-posedness of the closed-loop system and its asymptotical stability. For the asymptotic stability, in the sequel we will show that if we defne the energy of the system $\mathscr{S}$ as $E(t)=\frac{1}{2}\|z(t)\|_{X}^{2}$, where $z(t)$ is a solution of the system, then the rate of energy is given by (23), cf. (3). If we can apply LaSalle's invariance theorem, see (Luo, Guo and Morgül, 1999), then we can conclude that all solutions of system $\mathscr{S}$ asymptotically tend to the maximal invariant set contained in

$$
\mathscr{O}=\left\{z \in X \mid\langle z, \mathscr{A} z\rangle_{X}=0\right\} .
$$

Note that in the set $\mathscr{O}$, for any invariant solution we have $f_{i}^{j}(t)=0$, and for any $\alpha_{i}^{j}>0$ we also have $y_{i}^{j}(t)=0$ as well. If we can prove that, under these conditions the only possible solution of the system $\mathscr{S}$ is the zero solution, then by LaSalle's invariance theorem, we may conclude that all solutions of the system $\mathscr{S}$ asymptotically decay to zero. We note that in this case the inputs and the relevant outputs of the system $\mathscr{S}$ is zero, and the question of asymptotic stability is then related to the observability, see (Curtain and Zwart, 1995). 
By using (20) and (22) in (5), (6), we obtain

$$
\begin{aligned}
& \left(B_{i}^{1} w+\alpha_{i}^{1} O_{i}^{1} w_{t}\right)(0)=0, i=1, \ldots, k, \\
& \left(B_{i}^{2} w+\alpha_{i}^{2} O_{i}^{2} w_{t}\right)(1)=0, i=1, \ldots, l
\end{aligned}
$$

Let us consider the boundary conditions (25) and (26). To incorporate these in the closed-loop system, we defne the following set

$$
\begin{gathered}
\mathscr{S}_{1 c}=\left\{(u v)^{T} \in H \times H\right. \\
\left(B_{j}^{1} u+\alpha_{j}^{1} O_{j}^{1} v\right)(0)=0 \\
\left(B_{i}^{2} u+\alpha_{i}^{2} O_{i}^{2} v\right)(1)=0 \\
j=1, \ldots, k, \quad i=1, \ldots, l\}
\end{gathered}
$$

and defne $D\left(A_{c}\right)$ as

$$
D\left(A_{c}\right)=D(A) \cap \mathscr{S}_{2},
$$

where $\mathscr{S}_{2}$ is given by (8). By using the notation given above, the system $\mathscr{S}$ with the control law given by (22) can be rewritten as

$$
\frac{d z}{d t}=\mathscr{A} z, z(0) \in X
$$

where $X=V \times H$, the operator $\mathscr{A}$ is given by (15) and

$$
D(\mathscr{A})=\left(D\left(A_{c}\right) \times V\right) \cap \mathscr{S}_{1 c} .
$$

This system will be referred as the system $\mathscr{S}_{c}$. For this system we will make the following assumption. We would like to emphasize that this and the following assumptions should hold for all $\alpha_{i}^{j} \geq 0$.

Assumption 5 : The operator $\lambda I-\mathscr{A}: D(\mathscr{A}) \subset X \rightarrow$ $X$ is onto for all $\lambda>0$.

A simple consequence of this assumption is given in the following theorem.

Theorem 1 : Consider the system $\mathscr{S}_{c}$ given by (29) and let the Assumptions 1-5 hold. Then the operator $\mathscr{A}$ generates a $C_{0}$-semigroup of contractions $T(t)$ on $X$. If $z(0) \in D(\mathscr{A})$, then $z(t)=T(t) z(0)$ is the unique classical solution of (29) and $z(t) \in D(\mathscr{A})$ for $t \geq 0$. If $z(0) \in X$, then $z(t)=T(t) z(0)$ is the unique weak solution of (29).

Proof : The proof easily follows from the assumptions and the Lümer-Phillips Theorem, see (Pazy, 1983), (Luo, Guo and Morgül, 1999).

The following assumptions are required to establish some asymptotic stability results.

Assumption 6 : The operator $(\lambda I-\mathscr{A})^{-1}: X \rightarrow X$ is compact for $\lambda>0$.

Assumption 7 : The only invariant solution of (29) in the set $\mathscr{S}_{1} \cap \mathscr{S}_{2} \cap \mathscr{S}_{3}$ is the zero solution, where $\mathscr{S}_{1}$ and $\mathscr{S}_{2}$ are given by (7), (8) and $\mathscr{S}_{3}$ is given by

$$
\begin{gathered}
\mathscr{S}_{3}=\left\{(u v)^{T} \in H \times H \mid\right. \\
\quad\left(O_{i}^{1} v\right)(0)=0,\left(O_{j}^{2} v\right)(1)=0 \\
\quad \text { for } \alpha_{i}^{1}>0, i=1, \ldots, k, \text { for } \alpha_{j}^{2}>0, \\
j=1, \ldots, l\} .
\end{gathered}
$$

Theorem 2 : Let the assumptions 1-7 hold, consider the system $\mathscr{S}_{c}$ given by (29), and let $T(t)$ be the unique $C_{0}$-semigroup generated by $\mathscr{A}$. Then, the system $\mathscr{S}_{c}$ is globally asymptotically stable, that is for any $z(0) \in X$, the unique (clasical or weak) solution $z(t)=T(t) z(0)$ of (29) asymptotically approaches to zero, i.e. $\lim _{t \rightarrow \infty}\|z(t)\|_{X}=0$.

Proof : Proof follows from the assumptions and the LaSalle's invariance theorem, see (Luo, Guo and Morgül, 1999).

To establish the exponential stability, we may use the following well-known result.

Theorem 3 : Let the assumptions 1-5 hold, consider the system $\mathscr{S}_{c}$ given by (29), and let $T(t)$ be the unique $C_{0}$-semigroup generated by $\mathscr{A}$. Then $T(t)$ is exponentially stable, i.e. the following holds for some $M>0, \delta>0$

$$
\|T(t)\|_{X} \leq M e^{-\delta t}\|z(0)\|_{X},
$$

if and only if the following holds

$$
\sup _{\omega}\left\|(j \omega I-\mathscr{A})^{-1}\right\|_{X}<\infty
$$

Proof : This result is known as Huang's Theorem, see e.g. (Luo, Guo and Morgül, 1999)

In the applications, the diffcult part in using the Theorem 3 is to establish (33). Alternatively, we may use the so-called energy multiplier methods. One such result is given below.

Theorem 4 : Consider the system $\mathscr{S}_{c}$ given by (29) and let the assumptions 1-5 hold. Let $T(t)$ be the $C_{0^{-}}$ semigroup of contractions generated by $\mathscr{A}$. Let $z=$ $(u v)^{T} \in H$ and let us defne the projections $P_{1}: X \rightarrow$ $V, P_{2}: X \rightarrow H$ as $P_{1} z=u, P_{2} z=v$. Let $z(0) \in D(\mathscr{A})$ and let $z(t)$ denote the solution of (29). Assume that for a linear map $O: H \rightarrow H$ the following holds

$$
\begin{array}{r}
\left|<P_{2} z(t), O P_{1} z(t)>_{H}\right| \leq C E(t), \\
\frac{d}{d t}<P_{2} z(t), O P_{1} z(t)>_{H} \leq-E(t) \\
+\sum_{i=1}^{k} a_{i}^{1}\left(f_{i}^{1}\right)^{2}+\sum_{i=1}^{l} a_{i}^{2}\left(f_{i}^{2}\right)^{2},
\end{array}
$$

where $C>0$ and $a_{i}^{j}$ are arbitrary constants. Then the system $\mathscr{S}_{c}$ is exponentially stable, i.e. (32) holds.

Proof : See e.g. (Luo, Guo and Morgül, 1999) $\square$

The result given above can be used rather easily. However, note that this is only a suffcient condition, and that it may not be applicable to certain cases.

\section{AN EXAMPLE}

As an example, let us consider the following coupled wave equation

$$
u_{t t}-u_{x x}=\alpha(v-u), 0<x<1, t \geq 0,
$$




$$
\begin{gathered}
v_{t t}-v_{x x}=\alpha(u-v), 0<x<1, t \geq 0, \\
u(0, t)=0, u_{x}(1, t)=f(t), \\
v(0, t)=0, v_{x}(1, t)=g(t)
\end{gathered}
$$

see e.g. (Nafaji, 1992). Here, $\alpha>0$ is the coupling constant, $f(t)$ and $g(t)$ are the boundary control forces. We set $H=L_{2}(0,1) \times L_{2}(0,1)$. The operator $A: H \rightarrow H$ is defned as

$$
A\left(\begin{array}{l}
u \\
v
\end{array}\right)=\left(\begin{array}{l}
-u^{\prime \prime}-\alpha(v-u) \\
-v^{\prime \prime}-\alpha(u-v)
\end{array}\right) \text {. }
$$

Similar to previous example, we have

$$
D(A)=\left\{(u v)^{T} \in H \mid u, u^{\prime}, u^{\prime \prime}, v, v^{\prime}, v^{\prime \prime} \in H\right\} .
$$

Since $D(A)$ is dense in $H$, the Assumption 1 holds. The sets $\mathscr{S}_{1}$ and $\mathscr{S}_{2}$ can be found as

$$
\begin{aligned}
& \mathscr{S}_{1}=\left\{(u v)^{T} \in H \mid u(0)=v(0)=0\right\}, \\
& \mathscr{S}_{2}=\left\{(u v)^{T} \in H \mid u^{\prime}(1)=v^{\prime}(1)=0\right\} .
\end{aligned}
$$

Consequently, $D\left(A_{u c}\right)$ is found as

$$
\begin{aligned}
& D\left(A_{u c}\right)=\left\{(u v)^{T} \in H \mid(u v)^{T} \in D(A),\right. \\
& \left.u(0)=v(0)=0, u^{\prime}(1)=v^{\prime}(1)=0\right\} .
\end{aligned}
$$

For $z=(u v)^{T}$, we obtain

$$
\begin{aligned}
<z, A_{u c} z>_{H}= & \int_{0}^{1}\left[u\left(-u^{\prime \prime}-\alpha(v-u)\right)\right. \\
& \left.+v\left(-v^{\prime \prime}-\alpha(u-v)\right)\right] d x \\
= & \int_{0}^{1}\left(\left(u^{\prime}\right)^{2}+\left(v^{\prime}\right)^{2}\right) d x \\
& +\alpha \int_{0}^{1}(u-v)^{2} d x
\end{aligned}
$$

It can easily be shown that $A_{u c}$ is coercive, hence the Assumption 2 holds. As in previous example, we may choose $V$ as

$$
\begin{aligned}
V=D\left(A_{u c}^{1 / 2}\right)=\left\{(u v)^{T} \in H \mid\left(u^{\prime}, v^{\prime}\right)^{T} \in H,\right. \\
\left.u(0)=v(0)=0, u^{\prime}(1)=v^{\prime}(1)=0\right\}
\end{aligned}
$$

It then easily follows that the Assumption 3 is also satisfed. Accordingly we have $X=V \times H$ with the usual extension of the inner product in $L^{2}(0,1)$.

To show that the Assumption 4 is also satisfed, frst note that $D_{1}=D(A) \cap \mathscr{S}_{2}$ is dense in $D\left(A_{u c}\right)$. Let us set $z=\left(u v u_{1} v_{1}\right)^{T} \in X$, and $\tilde{z}$ similarly. From (41) it follows that the appropriate inner product in $X$ is the following :

$$
\begin{aligned}
<z, \tilde{z}>_{X}= & \frac{1}{2}\left(\int _ { 0 } ^ { 1 } \left(u \tilde{u}+v \tilde{v}+u_{1} \tilde{u}_{1}+v_{1} \tilde{v}_{1}\right.\right. \\
& +\alpha(u-v)(\tilde{u}-\tilde{v})) d x)
\end{aligned}
$$

By using the inner product given in (42), using integration by parts, after straightforward calculations we obtain the following

$$
<z, \mathscr{A} z>_{X}=u^{\prime}(1) u_{1}(1)+v^{\prime}(1) v_{1}(1)
$$

for any $z \in D_{1} \times V$. It then follows easily that the Assumption 4 is also satis£ed. Let $z=\left(u v u_{t} v_{t}\right)^{T} \in$ $D(\mathscr{A})$ be the solution of (37)-(39). Note that the Energy expression given by (19) becomes

$$
\begin{aligned}
E(t)= & \frac{1}{2}\left\langle z(t), z(t)>_{X}=\frac{1}{2}\left(\int _ { 0 } ^ { 1 } \left(u_{t}^{2}+v_{t}^{2}\right.\right.\right. \\
& \left.\left.+\left(u^{\prime}\right)^{2}+\left(v^{\prime}\right)^{2}+\alpha(u-v)^{2}\right) d x\right)
\end{aligned}
$$

Hence from (42)-(44) we obtain :

$$
\frac{d E}{d t}=f(t) u_{t}(1, t)+g(t) v_{t}(1, t)
$$

Therefore, the outputs $y_{1}$ and $y_{2}$ should be chosen as :

$$
y_{1}=u_{t}(1, t) \quad, \quad y_{2}=v_{t}(1, t)
$$

By using (22) we obtain :

$$
f(t)=-\alpha_{1} u_{t}(1, t), g(t)=-\alpha_{2} v_{t}(1, t) .
$$

By using (27) and (28) we obtain

$$
\begin{gathered}
\mathscr{S}_{1 c}=\left\{\left(z \in X \mid u^{\prime}(1)+\alpha_{1} u_{1}(1)=0,\right.\right. \\
\left.v^{\prime}(1)+\alpha_{2} v_{1}(1)=0\right\}, \\
D\left(A_{c}\right)=\{u \in D(A) \mid u(0)=v(0)=0\}
\end{gathered}
$$

Therefore, the system given above can be put into the form (29). Note that in this case $D(\mathscr{A})$ given by (30) becomes

$$
\begin{gathered}
D(\mathscr{A})=\left\{z \in X \mid(u v)^{T} \in D\left(A_{c}\right),\left(u_{1} v_{1}\right)^{T} \in V,\right. \\
u^{\prime}(1)+\alpha_{1} u_{1}(1)=0 \\
\left.v^{\prime}(1)+\alpha_{2} v_{1}(1)=0\right\}
\end{gathered}
$$

It can be shown that $\lambda I-\mathscr{A}: D(\mathscr{A}) \subset X \rightarrow X$ is onto for $\lambda>0$, see e.g. (Morgül, 1994). Hence, by Theorem $1, \mathscr{A}$ generates a $C_{0}$-semigroup of contractions on $X$. As in previous example, the Assumption 6 is also satis£ed. To prove the assumption 7 , let us assume that $\alpha_{1}>0$ and $\alpha_{2}=0$, i.e. only one boundary control force is active. In this case, the set $\mathscr{S}_{3}$ given by (31) is found as

$$
\mathscr{S}_{3}=\left\{z \in X \mid u_{1}=0\right\} \text {. }
$$

Hence accordingly we should look at the nonzero solutions of the system given by (36)-(39) with

$$
f(t)=0, g(t)=0, u_{t}(1, t)=0 .
$$

By using separation of variables, see e.g., (Meirovitch, 1967), we could fnd the possible solutions of this system. Note that, by using $w^{+}=u+v, w^{-}=u-$ $w$, this system of equations can be reduced to two decoupled system of equations of the form

$$
w_{t t}^{+}-w_{x x}^{+}=0, w^{+}(0)=0, w^{+\prime}(1)=0,
$$

$w_{t t}^{-}-w_{x x}^{+}+2 \alpha w^{-}=0, w^{-}(0)=0, w^{-1}(1)=0$.

It can be shown that the natural frequencies of the $\mathrm{frst}$ system are given by $\omega_{i}^{+}=\frac{(2 i+1) \pi}{2}, i=0,1, \ldots$ (i.e. the eigenvalues are $\left.\lambda_{i}=j \omega_{i}^{+}\right)$. Similarly, the natural frequencies of the second system are given by $\omega_{i}^{-}=$ 
$\sqrt{2 \alpha+\left(\omega_{i}^{+}\right)^{2}}$. By using these and the eigenvalue expansion, and noting that $2 u=w^{+}+w^{-}$, it follows that to have a nontrivial solution satisfying $u_{t}(1, t)=0$, for some $i$ and $j$, we must have $\omega_{i}^{+}=\omega_{j}^{-}$. Therefore, if this equation is not satisfed, then the only possible solution of this system is the trivial (i.e. zero) solution. Hence we conclude that if

$$
\alpha \neq \frac{1}{2}\left(\left(\omega_{i}^{+}\right)^{2}-\left(\omega_{j}^{-}\right)^{2}\right)
$$

for any $i, j$, then the system given above is asymptotically stable. It can also be shown that in this case exponential stability does not hold, and when $\alpha_{2}>0$ holds as well, this system is exponentially stable, see (Nafaji, 1992). We simulated this system for $\alpha_{1}=0$, $\alpha_{2}=0.1, \alpha=1$, and the simulation results are shown in the Figure 1. As can be seen, the asymptotic stability holds. Note that the same result holds for $\alpha_{1}>0$ and $\alpha_{2}=0$.

\section{CONCLUSION}

In this paper, we considered the feedback stabilization of a class of infnite dimensional systems by using boundary control, i.e. control inputs are applied at the boundaries of such systems. Such systems usually possess an internal energy, and along their solutions a conservation of energy equation hold. By utilizing this relation, we proved various stability results. The proposed approach could be used for the boundary stabilization of various conservative systems. Although we considered only static controllers in this work, the proposed approach could be generalized to the dynamic boundary controller case as well.

\section{REFERENCES}

Chen, G. (1979) Energy Decay Estimates and Exact Boundary Value Controllability for the Wave Equation in a Bounded Domain, J. Math. Pures. Appl., 58, pp.249-273.

Chen, G., M. C. Delfour, A. M . Krall and G. Payre (1987) Modelling, Stabilization and Control of Serially Connected Beams, SIAM J. Contr. Optimiz., 25, pp. 526-546.

Curtain, R. F., and H. J. Zwart (1995) An Introduction to In£nite Dimensional Linear Systems Theory, Springer Verlag, New York.

Kato, T. (1980) Perturbation Theory for Linear Operators, 2nd ed., Springer-Verlag, New York, .

Lions, J. L. (1988) Exact Controllability, Stabilization and Perturbations for Distributed Parameter Systems, SIAM Review, 30, pp. 1-68.
Luo, Z. H., B. Z. Guo, Ö. Morgül (1999) Stability and Stabilization of In£nite Dimensional Systems with Applications, Springer-Verlag, London.

Meirovitch, L. (1967) Analitical Methods in Vibration, New York : MacMillan.

Morgül, Ö. (1990) Control and Stabilization of a Flexible Beam Attached to a Rigid Body, Int. J. Contr., 51, pp. 11-33.

Morgül, Ö. (1991) Orientation and Stabilization of a Flexible Beam Attached to a Rigid Body : Planar Motion, IEEE Trans. on Auto. Contr., 36, pp. 953963.

Morgül, Ö. (1992) Dynamic Boundary Control of a Euler-Bernoulli Beam," IEEE Trans. on Auto. Contr., 37, pp. 639-642.

Morgül, Ö. (1994) “A Dynamic Boundary Control for the Wave Equation," Automatica, 30, pp. 17851792.

Nafaji, M., G. R. Sarhangi, and H. Wang, (1992) The study of the stabilizability of the coupled wave equations under various end conditions," Proceedings of the 31st CDC, Tucson, Arizona, pp. 374379.

Pazy, A. (1983) Semigroups of Linear Operators and Applications to Partial Differential Equations, Springer-Verlag, New York, .

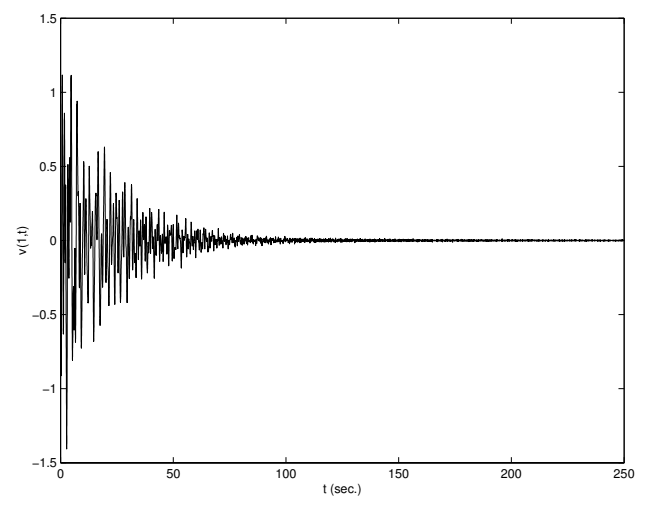

Fig. 1. Simulation result, $v(1, t)$ 